\title{
Improving safety and efficiency in care: multi-stakeholders' perceptions associated with a peritoneal dialysis virtual care solution
}

This article was published in the following Dove Press journal:

Patient Preference and Adherence

Lianne Jeffs, ${ }^{1-3}$ Trevor

Jamieson, ${ }^{4,5}$ Marianne

Saragosa, ${ }^{5}$ Geetha Mukerji, ${ }^{3,5,6}$

Arsh K Jain,, ${ }^{7}$ Rachel Man, ${ }^{7}$

Laura Desveaux, ${ }^{3,5}$ James

Shaw, ${ }^{3,5}$ Payal Agarwal, ${ }^{5,8}$

Jennifer M Hensel, ${ }^{5,9}$

Maria Maione, ${ }^{10}$ Megan

Nguyen, ${ }^{5}$ Nike Onabajo, ${ }^{5}$

R Sacha Bhatia ${ }^{3,5,11}$

'Sinai Health System, Toronto, ON M5G IX5, Canada; ${ }^{2} \mathrm{Li}$ Ka Shing Knowledge Institute, St Michael's Hospital, Toronto, ON M5G IWB, Canada; Institute of Health Policy, Management and Evaluation, University of Toronto, Toronto, ON M5S IB2, Canada; ${ }^{4}$ Department of Medicine, University of Toronto, Division of General Internal Medicine, St Michael's Hospital, Women's College Hospital, Toronto, ON M5G IWB, Canada; Institute for Health System Solutions and Virtual Care (WIHV), Women's College Hospital, Toronto, ON M5S IB2, Canada; 'Women's College Hospital, Toronto, ON M5S IB2, Canada; ${ }^{7}$ London Health Sciences Centre, London, ON N6A 5W9, Canada; ${ }^{8}$ Department of Family and Community Medicine, Women's College Hospital, University of Toronto, Toronto, ON M5S IB2, Canada; ${ }^{9}$ Department of Psychiatry, University of Toronto, Women's College Hospital, Women's College Research Institute, Women's College Hospital, Toronto, ON M5S IB2, Canada; ${ }^{10} \mathrm{St}$ Michael's Hospital, Toronto, ON M5G IWB, Canada; "University Health Network, Toronto, ON M5S IB2, Canada

Correspondence: Lianne Jeffs Sinai Health System, 600 University Avenue, Toronto, ON M5G IX5, Canada Email lianne.jeffs@sinaihealthsystem.ca
Background: Although there is a growing body of literature on the outcomes and impacts of remote home management with peritoneal dialysis (PD) patients, less is understood how this virtual care solution impacts the quality and efficiency of the healthcare system care. In this context, a study was undertaken to understand the perceptions of patients and their caregivers, healthcare providers, health system decision makers, and vendors associated with a remote monitoring and tracking solution aimed at enhancing the outcomes and experiences of chronic kidney disease (CKD) patients receiving PD at home.

Methods: A qualitative design using semi-structured interviews with 25 stakeholders was used in this study. Narrative data were analyzed by a thematic analysis approach.

Results: The following two themes emerged from the data: (1) leveraging data to monitor and intervene to keep patients safe and (2) increasing efficiencies and having control over supplies.

Discussion: Our study findings elucidated the ability of patients (and in some cases, caregivers) to monitor and trend their data and order and track directly on-line their dialysis supplies were key to their active participation in managing their CKD and keeping them safe at home. Their active participation and functionality of the virtual care solution also led to enhanced efficiencies (eg, process faster, easier, convenient, time savings) for both patients and healthcare providers Conclusion: The virtual care solution showed promising signs of a patient-centric approach and may serve as a blueprint for other virtual care solutions for chronic disease management. Keywords: peritoneal dialysis, chronic kidney disease, virtual care, qualitative research, patient centric care

\section{Introduction}

Chronic kidney disease (CKD) continues to affect over 70 million, or $10 \%$ of the adult population, worldwide ${ }^{1,2}$ and is associated with substantial costs to the healthcare system and society as a whole. ${ }^{3}$ In addition to these expenses, accessing kidney care to manage CKD can be challenging due to geographical barriers ${ }^{4,5}$ and a limited healthcare workforce with this area of clinical expertise. ${ }^{6-8}$ Accessing kidney care can place additional travel and lost employment costs and stress on patients (and their family members) that contribute to suboptimal care ${ }^{4}$ and increased mortality. ${ }^{5}$ Given the current costs and predicted increased costs associated with increased prevalence of CKD and the rising aging population with multiple comorbidities, ${ }^{1,4}$ different CKD care delivery models are required. ${ }^{9}$

A flexible model for managing CKD is providing peritoneal dialysis (PD) at home. There are promising signs that PD, estimated to be provided to 190,000 patients 
worldwide, ${ }^{10}$ and remote home management are providing a virtual care opportunity (telenephrology) ${ }^{11,12}$ for managing CKD. ${ }^{1}$ Telenephrology enables exchange of information and ongoing monitoring and surveillance of CKD patients' health statuses. ${ }^{12,13}$ Positive clinical and experiential outcomes (increased satisfaction, ${ }^{14,15}$ quality of life, ${ }^{16,17}$ survival, ${ }^{18-20}$ and decreased $\operatorname{costs}^{21}$ ) have been associated with PD. Similar outcomes have been reported with CKD home telemonitoring, including reductions in costs associated with in-center visits, hospital admissions, and transportation. ${ }^{4}$ Furthermore, PD patients on remote home management have reported reductions in hospital readmissions, emergency room visits, and lengths of stay in hospitals. ${ }^{1}$

Although there is a growing body of literature on the outcomes and impacts of remote home management with PD patients, less is understood around the perceptions of those involved in using virtual care technologies to support PD patients. ${ }^{12}$ Furthermore, an understanding from a variety of stakeholders is not known. In this context, a study was undertaken to gain insight into the perceptions of patients (and when present, their caregivers), healthcare providers, health system decision makers, and vendors associated with a virtual care solution aimed at enhancing the outcomes and experiences of CKD patients receiving $\mathrm{PD}$ at home.

\section{Methods}

\section{Study design}

The study design used was a qualitative approach which was conducted alongside a randomized, parallel-arm control trial aimed at determining if utilizing eQConnect ${ }^{\mathrm{TM}}$ (eQOL Inc., Marie, ON, CA) improves selected clinical outcomes for PD patients (CONNECT Trial). Prior to commencement of the qualitative study, ethics approval was obtained from participating sites (Women's College Hospital, St Michael's Hospital, London Health Sciences Center, and Humber River Regional Hospital) Research Ethics Boards.

\section{Virtual care solution description}

The virtual care solution (eQConnect ${ }^{\mathrm{TM}}$ ) developed by a vendor (eQOL Inc.) is telehomecare monitoring software that provides support for patients undergoing PD and includes a platform to exchange patient health information between patients and the healthcare team that aims to enhance patient compliance with PD regimens. Specifically, the platform consists of a patient-facing part (Patient Portal) that operates on a tablet (iPad Mini 2 running Apple iOS) and that is designed to record and upload data (eg, treatment progress, health status, supply usage) easily and securely over the Internet to a secure data. The clinician-facing part (Support Portal) enables clinicians to gain access to the data by logging in. A more detailed description of eQConnect ${ }^{\mathrm{TM}}$ is provided in a published protocol paper. $^{22}$

\section{Data collection and analysis}

Interviews with key stakeholders (patients, and when present, their caregivers; healthcare providers; health system decision makers; and vendors) involved in the implementation of eQConnect $^{\mathrm{TM}}$ virtual care solution were conducted. A semistructured interview guide was developed and used to ask questions about participants' perceptions and experiences of learning about and using the technology. Prior to the interview, a research assistant obtained verbal informed consent and conducted the telephone interview at a time convenient to study participants between baseline and 3 months of implementation of eQConnect ${ }^{\mathrm{TM}}$ from March to June 2017. The average length of time of interviews with patients/caregivers were 25 minutes (11-44 minutes); healthcare providers and vendors 22 minutes (8-44 minutes); and health system decision makers 49 minutes (44-59 minutes).

A thematic analysis approach was used to analyze the interviews. This process involved initially transcribing the interviews verbatim, which were then analyzed by thematic analysis. ${ }^{23}$ The transcribed interviews were then independently coded line-by-line to identify sections of text that serve as codes by two researchers. The researchers then met to determine the codes and categories through consensus and develop a coding schema that included themes and subthemes derived from the categorical data. As a final step, the principal investigator reviewed all transcripts to the emergent coding schema. For more details on the study methods, refer to the published protocol. ${ }^{22}$

\section{Results}

\section{Sample characteristics}

Twenty-five stakeholders (six patients and three caregivers; six healthcare providers; two vendors; and eight health system decision makers) participated in this study. In total, there were 21 interviews conducted with three patients being interviewed with their caregiver, three alone, and two health system decision makers interviewed together. In the patient and caregiver cohort, the average age was 65.6 (41-86) years; five were males and four were females; the majority were married ( $\mathrm{n}=5$ ), followed by common law (two), one single, and one divorced; seven were Caucasian with two being Asian and seven were educated at a university or college level and two at a high school level. The average length of time patients had been managing their CKD was 9.7 (1-31) years with the length of time being on dialysis predominately 
$1-3$ years $(n=7)$, followed by $3-5$ years $(n=1)$, and less than 1 year $(n=1)$. In the healthcare provider and vendor cohort, there were three project coordinators, two nurses, one physician, a product development manager, and a clinical coordinator. Within this cohort, five were females and three were males with three being in their current position 2-5 years; one 6-10 years; one 11-15 years; and over 16 years. No demographic information was obtained from the participating health system decision makers that drew from a variety of provincial agencies (eg, funder, networks).

\section{Themes}

Two themes emerged from the narrative dataset around the perceived value and outcomes associated with virtual care solution (eQConnect ${ }^{\mathrm{TM}}$ ). These include (1) leveraging data to monitor and intervene to keep patients safe and (2) increasing efficiencies and having control over supplies.

\section{Leveraging data to monitor and intervene to keep patients safe}

This theme captures how patients and providers leveraged data to monitor, trend, and intervene to keep patients safe. Patients, caregivers, and healthcare providers described how useful having timely (described by many participants as "real time") access to data (including uploading pictures) entered through the tablet was to monitor their health status (eg, treatment log, blood pressure, weights, bowel movements, etc.). This functionality enabled both patients and healthcare providers to "spot any trends" and to "keep track" of what was going on daily in between clinic visits that usually occurred every 3 months. Patients were also instructed by the CKD clinic team to contact the clinic if they suspected any adverse events or abnormalities in their health status. From this functionality of monitoring and trending, healthcare providers, upon their review of the data, identified deteriorating health status (eg, infections, higher blood pressure) and were able to intervene earlier. Interestingly, one patient described eQConnect ${ }^{\mathrm{TM}}$ as a "set of eyes" that healthcare providers can pick up on something that the patient might have missed. This theme is illustrated in the following quotes:

"They get the data on a regular basis rather than every 2 months so they have an ability to spot any trends. It's very useful getting timely data.” (Patient Site 1)

"At any time during any given day I can look at any of my patients and know exactly where they are and spot trends as a primary nurse. I can call them and say "I see you're doing really good" but I can also message them on the iPad and know exactly when they've read it." (Healthcare Provider Site 1)
"Patients can upload pictures if they're having a problem with the catheter exit site they can take a picture and our nurses can log in real time and see that picture come up. They can send messages back and forth in real time and the nurses are able to track and see treatment records, so they can see all the blood pressures in real time and they can track it and see any trend that's happening or any weight issues. Patients do not have to wait 2 months before they can show the nurse and healthcare team." (Healthcare Provider Site 2)

Keeping patients safe included preventing error and harm and alleviating anxiety and increasing comfort associated with having and managing $\mathrm{CKD}$. Both healthcare providers and decision makers described the impact of the functionality of eQConnect ${ }^{\mathrm{TM}}$ including the decreased progression of infections, better blood pressure control, and reduced hospitalization and clinic visits. The use of eQConnect also enabled the healthcare provider to address deficiencies in patient selfmanagement (eg, inability to identify early infection or nonadherence to treatment regime). Furthermore, one healthcare provider described that in some cases referral of patients to the emergency department was an appropriate intervention to ensure patients did not get worse. This first theme is further elucidated in the following narrative excerpts.

"We brought a patient in with an exit site infection that we treated quickly and we actually found it because of the iPad. She wrote in information that was a change on her exit site and on seeing that change we called her and brought her in and she did have an infection which we, we we're treating that before it became a serious situation. We treated her faster than she actually would have called." (Healthcare Provider Site 1)

"One patient was losing dwell time so he was not getting the full treatment, he kept cutting his treatment short for some reason and that's something that's inputted into the iPad so the nurses were seeing that. Based on that they were able to make an adjustment and were able to resolve that." (Healthcare Provider Site 2)

"When they need it to help them with the anxiety of managing in home dialysis you know from a clinicians perspective to be able to provide that ongoing monitoring at the convenience of the patient and not having to do inperson visits and also having some automated monitoring." (Health System Decision Maker)

"It's incredibly beneficial for a number of reasons, I mean the communication aspects are amazing, the ability to monitor and view patients, especially patients that are having problems with their dialysis or are recovering from infections." (Vendor 2) 


\section{Increasing efficiencies and having control over supplies}

This theme captured how eQConnect ${ }^{\mathrm{TM}}$ resulted in increased efficiencies for the patient, the provider, and the healthcare system. Efficiencies described by study participants included making the process easier, convenient, faster, accurate and efficient; demonstrating work-flow; and cost savings and having control over ordering dialysis supplies. Patients shared that they were satisfied with using eQConnect ${ }^{\mathrm{TM}}$ and recommended its use for other CKD patients. Several of the patients and healthcare providers described that using the tablet made the process of entering data and monitoring their treatment and ongoing health status more efficient, convenient, faster, accurate, easier, and simpler. Healthcare providers and health system decision makers also described using the eQConnect ${ }^{\mathrm{TM}}$ led to a more efficient workflow that can result in cost savings to the patient and the system. The following narrative excerpts illustrate this theme:

"It's better than having to write everything down. It's easily accessible. When I used to have to use the binder and write it all down. Half the time I would not even take my blood pressure, I got lazy. It's faster. I think everybody should have one. It makes it easy for me to do my reporting and stuff, but it really as far as how I feel, it really does not control that at all." (Patient Site 1)

“The way the iPad is designed, it's so simple, it's so easy for them to manage and once they get through that little bump of knowing okay what I'm doing is right and seeing that its going through to us and we're acknowledging everything is coming through to us - its a good tool because they know that we are connecting to them daily." (Healthcare Provider Site 1)

"From a system perspective you know if we can get patients from hospital dialysis into in home dialysis, there's a huge cost saving for the healthcare system." (Health System Decision Maker)

Within this theme, healthcare providers and health system decision makers described the value of patients and their caregivers being able to have control over ordering their supplies for dialysis at home. Healthcare providers highlighted this aspect as a unique feature compared to other patient monitoring solutions and the vendor highlighted that there was also a function that hospitals could use to track supplies and associated costs. The functionality of the tablet served as an inventory control that provided an overview of what supplies are needed and reminders of when to order as noted in the following narrative excerpts:

"I was a little nervous, and it worked just fine because I was used to calling in and having them call me once a month and the lady would go through the supply list with me. Now I enter that into the computer the supplies that you have use. The inventory control is wonderful. That part of it is great because on order day I just go through and do a count of what I have and match it up to what's on the iPad and usually it's bang on." (Patient Site 1)

"They can keep track of their supplies - they can order their supplies on it." (Healthcare Provider Site 1)

"Beyond that in the software they have a calendar so for this we program in their supply order dates and delivery dates automatically so they reduce the frequency that they miss those dates and have to get emergency deliveries. Beyond that they have the option of ordering their supplies through the software, so this helps the nurses look at what they're using, identify maybe any misusage if they're overordering on different things. It creates a tracking system for where the supplies are going because that's a huge cost for the hospital as a whole." (Vendor)

\section{Discussion}

Our study findings delineate the perceived outcomes associated with a virtual care solution offered to patients receiving PD at home. Specifically, our first theme elucidates how the virtual care solution enables patients (and in some cases their family caregivers) to actively participate in their care by inputting data that both they and members of the CKD clinic team used to monitor their health statuses. Having the eQConnect ${ }^{\mathrm{TM}}$ as part of patients' care processes alleviated anxiety around managing their $\mathrm{CKD}$ at home. Telemonitoring enables patients to actively participate in effectively co-managing their CKD and improves their autonomy and adherence to their care plan as they better understand how best to control their chronic disease and disease-related symptoms. ${ }^{1,4,24}$

In our study, patients and members of the CKD clinic team were able to identify a deteriorating status (eg, infection, high blood pressure) using the remote telemonitoring function. This finding is consistent with other literature that highlights that telemonitoring enables rapid detection of early symptoms that signal potential deterioration of a patient's health status. ${ }^{4,13}$ In turn, the ability to identify the deteriorating status was reported to prevent complications 
and reduce visits to the clinic or hospital is consistent with other literature. ${ }^{1,4,9,24,25}$

Our theme around how the use of eQConnect ${ }^{\mathrm{TM}}$ resulted in enhanced efficiencies for both patients and healthcare providers adds to a growing body of literature. Specifically, our finding around $\mathrm{eQConnect}{ }^{\mathrm{TM}}$ being more efficient, easier, and convenient for patients to use is echoed by others. ${ }^{4,9,26} \mathrm{In}$ our study, patients described how eQConnect ${ }^{\mathrm{TM}}$ became part of their daily care routine. The finding around recommending eQConnect ${ }^{\mathrm{TM}}$ to other CKD patients is similar to another study. ${ }^{27}$ The perceived cost savings due to reduced travel and healthcare costs associated with clinic and hospital visits reported in our study is consistent with the literature. ${ }^{4,28}$ This literature describes the use of home telemonitoring and how it contributes to cost containment by limiting the need for patient transportation, reducing the number of clinic visits, hospitalizations, and preventing complications by detecting and addressing deteriorating health status. ${ }^{4,27}$ In our study, healthcare providers also viewed eQConnect ${ }^{\mathrm{TM}}$ as easy to use, efficient, and embedded into their daily workflow. This finding provides empirical support for feasibility of embedding virtual care solutions into the daily workflow of healthcare providers. ${ }^{29,30}$

The functionality of inventory management of dialysis supplies emerged as a unique finding in this study. Specifically, the ability of patients (and in some cases, caregivers) to be able to order and track directly on-line their dialysis supplies was key to their active participation and managing their CKD at home. Although specific literature around inventory management using a virtual care solution for patients with CKD receiving PD at home was not located, our study findings provide an example of truly "patient-centric" care (ie, a solution targeting a highly patient-focused task or need). Virtual care solutions are one means of making the healthcare system more "patientcentric" and "relationship-based care" whereby care is complementary and personalized providing new ways for patients and healthcare providers within existing relationships to interact. ${ }^{1,26,29}$ Further, patient-centric care is responsive to patients' needs and more convenient and accessible for patients. ${ }^{26,29} \mathrm{~A}$ core component of patient-centric care is patient empowerment that enables patient independence, self-management, and self-efficacy. ${ }^{31}$ Empowerment promotes equitable and collaborative approaches to healthcare and improved cost-effectiveness of care delivery. ${ }^{32}$ There is also the potential for improving utilization and outcomes by incorporating functions that are more directly and immediately incentivizing to users-a core principle of usercentered design. ${ }^{33}$

There are limitations inherent in our design including the transferability of the qualitative data to other types of healthcare organizations due to the study being conducted at two hospitals in Southern Ontario. There are also selection biases that are inherent in study participants volunteering to participate including those participating in an intervention trial study of which the patients who participated were recruited from. There was no comparison group to compare and contrast the experiences of using (or not) the virtual care solution which further limits the transferability of findings (which were positive in nature) to other settings. Further, only one physician from one site was recruited to participate in the interviews.

Due to delays in obtaining ethics approvals, the data collection period for this evaluation was limited to a 4-month period of which there are a limited number of patients enrolled at each site to recruit into the study. Interviews were conducted during a 3-month window, implying varying levels of familiarity with the application at the time of the interview; however, we do not feel this would bias our results and feel that interviewing parties with varying experience is important to capture issues relating to early usage while mitigating the impact of the learning curve. Although we anticipate that there might be a decline in usage of the virtual care solution over time, we were not able to capture this due to the short period for data collection. Furthermore, the limited number of subjects per category of stakeholders and the absence of data on the real impact on the safety, quality, and efficiency of the healthcare system care, inhibits deriving any strong clinical, organizational and managerial implications about the virtual care solution. Future research efforts need to collect and examine data on the "real impact" with a focus on the efficacy, efficiency, and viability of the virtual care solution.

\section{Conclusion}

Currently, limited empirical evidence exists on how patients view virtual care and how it affects overall health system use. ${ }^{26}$ Our study elucidated the perspectives of patients, caregivers, healthcare providers, health system decision makers, and vendors on a virtual care solution aimed at enhancing the outcomes and experiences of CKD patients receiving $\mathrm{PD}$ at home. Collectively, the features of the virtual care solution (data repository and surveillance monitoring, inventory management, and communication tool) explored in this study exemplified a patient-centric virtual care solution 
that showed the promise of improving safety and efficiency. Inventory management, a feature targeting a highly patientcentric priority, may serve as a blueprint for other virtual care solutions to improve user engagement with chronic disease management. Further research is required to explore and examine these possibilities.

\section{Acknowledgment}

The authors would like to acknowledge the Ontario Telemedicine Network for their engagement and operational oversight as well as Canada Health Infoway and the Ministry of Health and Long Term Care.

\section{Disclosure}

The authors report no conflicts of interest in this work.

\section{References}

1. He T, Liu X, Li Y, Wu Q, Liu M, Yuan H. Remote home management for chronic kidney disease: a systematic review. J Telemed Telecare. 2017;23(1):3-13.

2. Jha V, Garcia-Garcia G, Iseki K, et al. Chronic kidney disease: global dimension and perspectives. Lancet. 2013;382(9888):260-272.

3. Vanholder R, Annemans L, Brown E, et al. Reducing the costs of chronic kidney disease while delivering quality health care: a call to action. Nat Rev Nephrol. 2017;13(7):393-409.

4. Thilly N, Chanliau J, Frimat L, et al. Cost-effectiveness of home telemonitoring in chronic kidney disease patients at different stages by a pragmatic randomized controlled trial (eNephro): rationale and study design. BMC Nephrol. 2017;18(1):126.

5. Rucker D, Hemmelgarn BR, Lin M, et al. Quality of care and mortality are worse in chronic kidney disease patients living in remote areas. Kidney Int. 2011;79(2):210-217.

6. Ong SW, Jassal SV, Miller JA, et al. Integrating a smartphone-based self-management system into usual care of advanced CKD. Clin J Am Soc Nephrol. 2016;11(6):1054-1062.

7. Bello AK, Hemmelgarn B, Lin M, et al. Impact of remote location on quality care delivery and relationships to adverse health outcomes in patients with diabetes and chronic kidney disease. Nephrol Dial Transplant. 2012;27(10):3849-3855.

8. Liddy C, Rowan MS, Afkham A, Maranger J, Keely E. Building access to specialist care through e-consultation. Open Med. 2013;7(1): e1-e8.

9. Bello AK, Molzahn AE, Girard LP, et al. Patient and provider perspectives on the design and implementation of an electronic consultation system for kidney care delivery in Canada: a focus group study. BMJ Open. 2017;7(3):e014784.

10. Jain AK, Blake P, Cordy P, Garg AX. Global trends in rates of peritoneal dialysis. J Am Soc Nephrol. 2012;23(3):533-544.

11. Gordon EJ, Fink JC, Fischer MJ. Telenephrology: a novel approach to improve coordinated and collaborative care for chronic kidney disease. Nephrol Dial Transplant. 2013;28(4):972-981.

12. Osman MA, Okel J, Okpechi IG, Jindal K, Bello AK. Potential applications of telenephrology to enhance global kidney care. BMJ Glob Health. 2017;2(2):e000292.
13. Weiner S, Fink JC. Telemedicine to promote patient safety: use of phonebased interactive voice-response system to reduce adverse safety events in pre-dialysis CKD. Adv Chronic Kidney Dis. 2017;24(1):31-38.

14. Rubin HR, Fink NE, Plantinga LC, Sadler JH, Kliger AS, Powe NR. Patient ratings of dialysis care with peritoneal dialysis vs hemodialysis. J Am Med Assoc. 2004;291(6):697-703.

15. Li J, Wang H, Xie H, et al. Effects of post-discharge nurse-led telephone supportive care for patients with chronic kidney disease undergoing peritoneal dialysis in China: a randomized controlled trial. Perit Dial Int. 2014;34(3):278-288.

16. Griva K, Yu Z, Chan S, et al. Age is not a contraindication to home-based dialysis - Quality-of-Life outcomes favour older patients on peritoneal dialysis regimes relative to younger patients. $J$ Adv Nurs. 2014;70(8): 1902-1914.

17. Brown E. Peritoneal dialysis: older patients report better quality of life than younger. Evid Based Nurs. 2015;18(3):93-93.

18. Kaldoudi E, Passadakis P, Panagoutsos S. Homecare telematics for peritoneal dialysis. J Inform Techn Healthcare. 2004;5:372-378.

19. Chaudhary K, Sangha H, Khanna R. Peritoneal dialysis first: rationale. Clin J Am Soc Nephrol. 2011;6(2):447-456.

20. Murphy SW, Foley RN, Barrett BJ, et al. Comparative mortality of hemodialysis and peritoneal dialysis in Canada. Kidney Int. 2000: 571720-571726.

21. Chui BK, Manns B, Pannu N, et al. Health care costs of peritoneal dialysis technique failure and dialysis modality switching. Am J Kidney Dis. 2013;61(1):104-111.

22. Jeffs L, Jain AK, Man RH, Tang RHT, et al. Exploring the utility and scalability of a telehomecare intervention for patients with chronic kidney disease undergoing peritoneal dialysis-a study protocol. $B M C$ Nephrol. 2017;18(1):155.

23. Braun V, Clarke V. Using thematic analysis in psychology. Qual Res Psychol. 2006;3(2):77-101.

24. Chen AH, Murphy EJ, Yee HF, Jr. eReferral - a new model for integrated care. N Engl J Med. 2013;368(26):2450-2453.

25. Gallar P, Vigil A, Rodriguez I, et al. Two-year experience with telemedicine in the follow-up of patients in home peritoneal dialysis. J Telemed Telecare. 2007;13(6):288-292.

26. Mcgrail KM, Ahuja MA, Leaver CA. Virtual visits and patient-centered care: results of a patient survey and observational study. $J$ Med Internet Res. 2017;19(5):e177.

27. Fink JC, Doerfler RM, Yoffe MR, et al. Patient-reported safety events in chronic kidney disease recorded with an interactive voice-inquiry dial-response system: monthly report analysis. J Med Internet Res. 2016;18(5):e125.

28. Ishani A, Christopher J, Palmer D, et al. Telehealth by an interprofessional team in patients with CKD: a randomized controlled trial. Am J Kidney Dis. 2016;68(1):41-49.

29. Boston-Fleischhauer C. The explosion of virtual nursing care. $J$ Nurs Adm. 2017;47(2):85-87.

30. Shaw J, Jamieson T, Agarwal P, et al. Virtual care policy recommendations for patient-centred primary care: findings of a consensus policy dialogue using a nominal group technique. J Telemed Telecare. 2018; 24(9): 17730444.

31. Risling T, Martinez J, Young J, Thorp-Froslie N. Evaluating patient empowerment in association with eHealth technology: scoping review. J Med Internet Res. 2017;19(9):e329.

32. Mcallister M, Dunn G, Payne K, Davies L, Todd C. Patient empowerment: the need to consider it as a measurable patient-reported outcome for chronic conditions. BMC Health Serv Res. 2012;12:157.

33. Mccurdie T, Taneva S, Casselman M, et al. mHealth consumer apps: the case for user-centered design. Biomed Instrum Technol. 2012;46(s2):49-56. 
Patient Preference and Adherence

Dovepress

\section{Publish your work in this journal}

Patient Preference and Adherence is an international, peer-reviewed, open access journal that focuses on the growing importance of patient preference and adherence throughout the therapeutic continuum. Patient satisfaction, acceptability, quality of life, compliance, persistence and their role in developing new therapeutic modalities and compounds to optimize

Submit your manuscript here: http://www.dovepress.com/patient-preference-and-adherence-journ clinical outcomes for existing disease states are major areas of interest for the journal. This journal has been accepted for indexing on PubMed Central. The manuscript management system is completely online and includes a very quick and fair peer-review system, which is all easy to use. Visit http://www. dovepress.com/testimonials.php to read real quotes from published authors. 